\title{
Flexible drift-compensation system for precise 3D force mapping in severe drift environments
}

\author{
Philipp Rahe, ${ }^{1, a)}$ Jens Schütte, ${ }^{1, b)}$ Werner Schniederberend, ${ }^{2}$ Michael Reichling, ${ }^{3}$ \\ Masayuki Abe, ${ }^{4}$ Yoshiaki Sugimoto, ${ }^{4}$ and Angelika Kühnle ${ }^{1}$ \\ ${ }^{1}$ Institut für Physikalische Chemie, Fachbereich Chemie, Johannes Gutenberg-Universität Mainz, \\ Jakob-Welder-Weg 11, 55099 Mainz, Germany \\ ${ }^{2}$ Werkstatt für Elektronik und Informationstechnik, Universität Osnabrück, Barbarastrasse 7, \\ 49076 Osnabrück, Germany \\ ${ }^{3}$ Fachbereich Physik, Universität Osnabrück, Barbarastrasse 7, 49076 Osnabrück, Germany \\ ${ }^{4}$ Graduate School of Engineering, Osaka University, 2-1 Yamada-Oka, 565-0871 Suita, Osaka, Japan
}

(Received 10 March 2011; accepted 19 May 2011; published online 22 June 2011)

\begin{abstract}
The acquisition of dense 3D data sets is of great importance, but also a challenge for scanning probe microscopy (SPM). Thermal drift often induces severe distortions in the data, which usually constrains the acquisition of dense data sets to experiments under ultra-high vacuum and low-temperature conditions. Atom tracking is an elegant approach to compensate for thermal drift and to position the microscope tip with highest precision. Here, we present a flexible drift compensation system which can easily be connected to existing SPM hardware. Furthermore, we describe a 3D data acquisition and position correction protocol, which is capable of handling large and non-linear drift as typically present in room temperature measurements. This protocol is based on atom-tracking for precise positioning of the tip and we are able to acquire dense 3D data sets over several hours at room temperature. The performance of the protocol is demonstrated by presenting 3D data taken on a $\mathrm{CaCO}_{3}(10 \overline{1} 4)$ surface with the data density being as large as $85 \times 85 \times 500$ pixel. () 2011 American Institute of Physics. [doi:10.1063/1.3600453]
\end{abstract}

\section{INTRODUCTION}

Atomic force microscopy (AFM) has proven to be a powerful tool in surface science, with the non-contact (NC) mode now providing atomic resolution on a routine basis. ${ }^{1,2}$ The acquisition of three-dimensional (3D) data, specifically 3D force fields above a surface, is of especial importance in numerous fields including surface chemistry, thin-film growth, molecular electronics, and nanotribology. ${ }^{3}$ Recently, the identification of the chemical structure of a pentacene molecule has been demonstrated by recording a complete 3D data set. ${ }^{4}$ Moreover, the short-range chemical interaction on surfaces such as $\mathrm{Si}(111),{ }^{5,6} \mathrm{KBr}(001),{ }^{7-9} \mathrm{NaCl}(100),{ }^{10,11}$ $\mathrm{NiO}(001),{ }^{12-14} \mathrm{MgO} / \mathrm{Ag}(001),{ }^{15}$ and graphite ${ }^{16}$ has been investigated at the atomic level by means of $3 \mathrm{D}$ NC-AFM force mapping. Furthermore, 3D data have been taken on small molecules adsorbed on metal substrates such as PTCDA/Cu(111) ${ }^{17}$ and PTCDA/Ag $(111)^{18}$ and on large structures such as carbon nanotubes. ${ }^{19}$

In most of these experiments either single images or single line data (spectroscopy data) have been combined to form a 3D data set with the aid of routines for distortion correction. ${ }^{20,21}$ Distortion correction is usually necessary, as (thermal) drift results in an unwanted movement between tip and sample. However, most correction procedures imply assumptions about the surface structure which might not be justified for all sample systems. Therefore, undistorted exper-

\footnotetext{
a) Electronic mail: rahe@uni-mainz.de.

b) Now at Dr. Eberl MBE-Komponenten GmbH, Gutenbergstraße 8, 71263 Weil der Stadt, Germany.
}

imental data are of particular interest. Consequently, so far all of the experiments with a large and dense number of data points (and, therefore, with a long acquisition time) have been carried out in a low-temperature environment as this reduces thermal drift velocities by orders of magnitude. Therefore, the residual thermal drift is much easier to compensate - which is in sharp contrast to a room-temperature environment, where thermal drift is usually large and non-linear. ${ }^{8-10,17,18}$ Using scan protocols based on consecutive image or line data acquisition, the densest 3D data set presented so far consists of $256 \times 256 \times 140$ pixel (total of 9175040 pixel) acquired during $40 \mathrm{~h}$ in a low-temperature environment, ${ }^{16}$ where, however, intense data processing routines were necessary for subsequent drift correction. In contrast, from room-temperature experiments only a maximum number of $34 \times 10 \times 481$ pixel (total of 163540 pixel) acquired during 15 min has been presented. ${ }^{10}$ In a liquid environment, the high-speed acquisition of $64 \times 64 \times 155$ pixel (total of 634880 pixel) in 53 s has been demonstrated ${ }^{22}$ which, however, demanded highly optimized scan electronics. ${ }^{23}$

Atom tracking ${ }^{24}$ is an elegant technique to measure thermal drift with high precision. It has initially been introduced to monitor fast diffusion processes. ${ }^{25-29}$ Abe et al. ${ }^{30-32}$ subsequently adapted this principle for drift measurement and compensation. The drift compensation in their case is performed using a feed-forward technique, which relies on the previously determined drift vector. Furthermore, the atom-tracking principle has been utilized by the same group for reproducible line data acquisition in a large-drift room-temperature environment. ${ }^{30} \mathrm{~A}$ comparable approach to the protocol presented later in this work has been implemented with a 
commercial scan controller system very recently, where the acquisition of $71 \times 71 \times 256$ pixel (total of 1290496 pixel) has been achieved over nearly $24 \mathrm{~h}$ with atom-tracking drift correction. ${ }^{11}$

Here, we present a new drift compensation system, based around highly flexible electronics and software. This flexibility allows this system to be used with any SPM scan electronics. We implement the atom-tracking and feed-forward techniques for high-precision drift velocity measurement and compensation, in addition to high-precision tip positioning. Furthermore, we describe an automated scan protocol for the acquisition of 3D data sets, yielding reliable data acquired under large and non-linear drift conditions. This protocol relies on alternating data acquisition and drift vector updates. In contrast to several other methods presented in literature, the acquired data do not require any subsequent distortion correction. This protocol is implemented in our drift compensation system and its performance is demonstrated by presenting $3 \mathrm{D}$ data acquired on a calcite $\mathrm{CaCO}_{3}(10 \overline{1} 4)$ surface.

The paper is organized as follows: in Sec. II the atomtracking principle is reviewed, followed in Sec. III by the description of our implementation. The characteristics of our system including noise figures are analyzed in Sec. IV. Our automated protocol for the 3D data acquisition under large and non-linear drift conditions is described in Sec. V and we demonstrate the performance of this acquisition protocol in Sec. VI by showing results obtained on a calcite $\mathrm{CaCO}_{3}(10 \overline{1} 4)$ surface.

\section{ATOM-TRACKING PRINCIPLE}

For atom tracking, ${ }^{24}$ the tip of a scanning probe microscope is dithered on the sample surface, describing a circle with radius $A_{\text {osc }}$ and frequency $f_{\text {osc }}$ above a circular surface feature, e.g., a single atom. The distance feedback loop of the scan controller is usually activated with a bandwidth setting larger than $f_{\text {osc }}$. If a radially symmetric feature with a lateral extension in the order of $A_{\text {osc }}$ is present right in the middle of the dither circle, the circular tip movement lies in a plane parallel to the surface. However, if the tip is slightly displaced from the feature center, it follows an equiline of interaction, which is no longer in a plane parallel to the surface. The vertical deviations $\delta z_{x}$ and $\delta z_{y}$ of the tip trajectory along both lateral directions can be decomposed by a two-phase lockin amplifier (LIA) into the in-phase (X) and quadrature (Y) signal, providing a measure for the lateral tip misplacement from the center of the feature. ${ }^{24,30}$ Using two independent feedback loops with the initial conditions $\delta z_{x}=\delta z_{y}=0$, the tip is locked to the feature and its movement can be monitored via the error signals of the feedback loops. Now, either fast surface diffusion of the feature or, if the tracked feature is immobile on the sample surface, the (thermal) drift movement can be monitored. The long-time behavior of the drift movement is usually extrapolated by a linear model in a feedforward routine. Hence, linear thermal drift is compensated by adding time-varying signals to the scan waveforms.

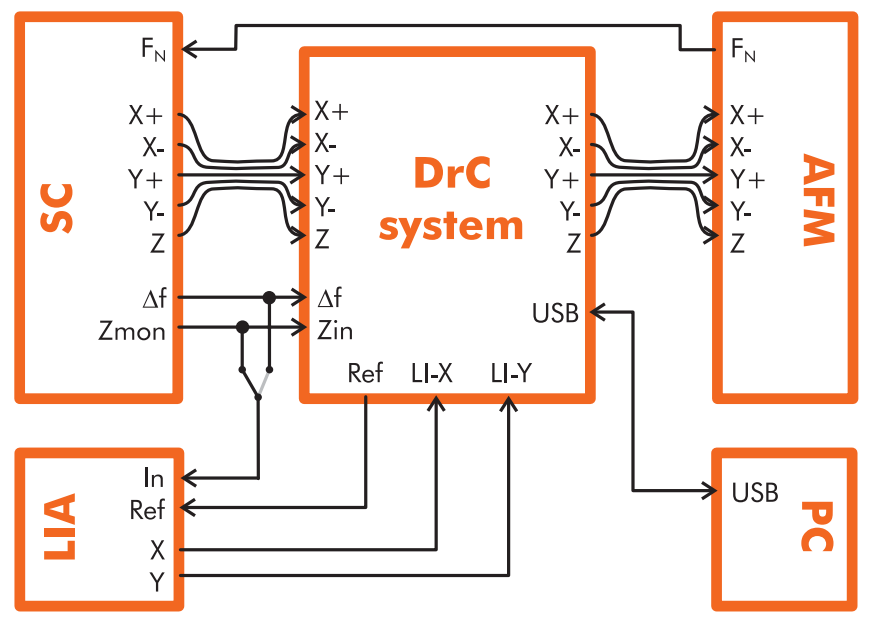

FIG. 1. (Color online) Connections of the drift-compensation (DrC) system with the scan controller (SC), the atomic force microscope (AFM), the lockin amplifier (LIA), and the computer (PC). The five piezo signals $X \pm, Y \pm, Z$ are high-voltage signals whereas all other connections are low-voltage inputs and outputs. $F_{\mathrm{N}}$ is the cantilever deflection signal.

\section{IMPLEMENTATION}

The tip of an SPM is usually positioned by piezo elements. The voltages inducing the piezo deflections are generated by means of the scan controller (SC), usually including a high-voltage amplification as its last stage. The physical movement of the tip and its positioning precision is directly related to the SC signal generation quality and amplification quantity. The central idea of our drift compensation implementation is to loop the signals for drift compensation in the high-voltage signal path from the SC to the scanner piezos, which is depicted schematically in Figure 1. This strategy is preferred to adding low-voltage signals inside the SC. The low-noise summation circuitry installed inside the drift compensation system will be described later in this section. Most of the system's flexibility, however, follows from this circuitry.

Additionally, several low-voltage input and output channels are available to connect signals such as the $\mathrm{X}, \mathrm{Y}$ and reference with the LIA as well as the detuning $(\Delta f)$ feedback channel and the topography $(Z)$ signal generated by the SC. Finally, the system communicates with a controlling computer via a USB connection. In Figure 1, two possibilities are drawn connecting the LIA input signal: either the $\mathrm{Z}$ channel or the $\Delta f$ feedback signal can be used. Which signal is most suitable relies on the quality of each and, furthermore, care has to be taken when setting the bandwidth of the distance feedback loop.

The drift compensation system itself makes use of a microcontroller (ATMega2560 from Atmel, San Jose CA, USA) for data flow control, status handling, and dynamic procedure generation. For the analog-to-digital conversion, $10 \mathrm{bit}$ and 20 bit analog-to-digital converters are used (internal ATMega2560 converter and LTC2420 from LinearTechnology, Milpitas CA, USA), depending on the required resolution and sampling time. The signal generation of each output channel is realized by external 16 bit digital-to-analog converters 


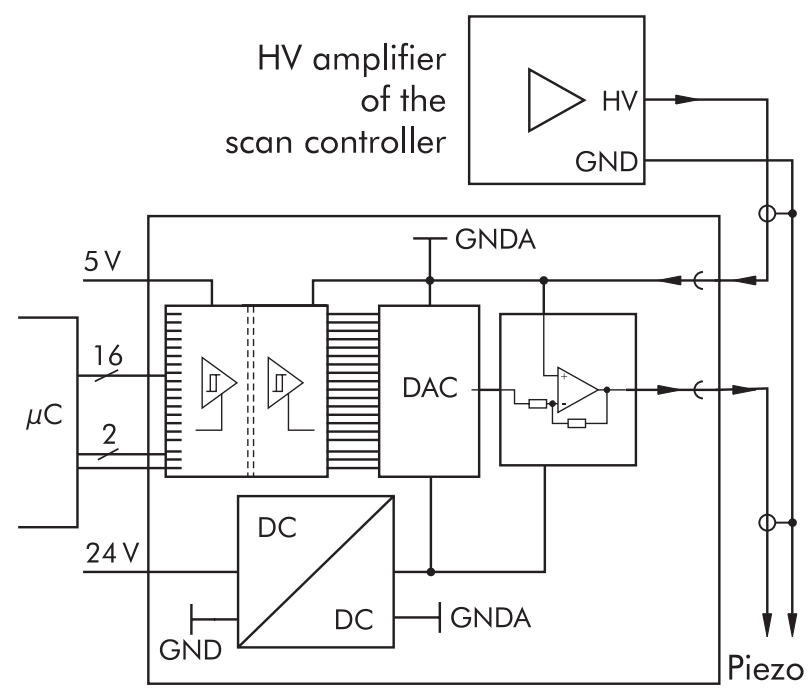

FIG. 2. Summation circuitry used for each single output channel. The DAC and the low-voltage OPA are decoupled from ground, thus allowing for the summation of the low-voltage drift compensation signals to the high-voltage SC signals.

(DAC) (DAC712 from Texas Instruments, Dallas TX, USA). Their wiring will be discussed in more detail later in this section. The microcontroller itself is connected to a controlling computer by a serial interface emulated on a USB connection. The program for the microcontroller is written in $\mathrm{C}$ and compiled using the AVR-GCC software development tools. ${ }^{33}$ It features real-time capability using timers and interrupt routines, required for oscillation signal generation, feedback loop control, and the feed-forward module. Most of the code's functionality is implemented as a finite state machine with the controlling computer inducing the state transitions. On the computer, a LABVIEW program is used, implementing the communication with the microcontroller as well as the data handling and the data representation.

The critical part of the system is the summation of the drift compensation signals with the SC signals. The magnitude of the latter signals is typically a few hundred volts while for the drift compensation, signals of a smaller voltage with increased precision are adequate. To meet our demands of low noise and maximum flexibility, we utilize a summation circuitry with a galvanic isolation of the DAC and operational amplifiers (OPA) from common ground, as is depicted in Figure 2. With this principle, we can use low-voltage operational amplifiers with low-noise characteristics for the high-precision summation of the drift compensation signals to the high-voltage SC signals. Furthermore, the summation circuitry does not demand any changes in the scan controller or microscope but can easily be connected to any SPM system.

The isolation is designed for input voltages of up to $1 \mathrm{kV}$. The decoupling of the digital signal lines is realized by digital isolators (ADuM1400 from Analog Devices, Norwood MA, USA), while DC/DC converters (THI2M and TES1 from TracoPower, Zürich, Switzerland) are used for the power supply. The residual resistive and capacitive coupling is at least $10 \mathrm{G} \Omega$ and at most $20 \mathrm{pF}$, respectively, and does not induce any significant stray couplings.

\section{NOISE CHARACTERIZATION AND PERFORMANCE}

The drift-compensation system is connected to a VT AFM 25 microscope from Omicron (Taunusstein, Germany) operated in the non-contact frequency modulation mode. The scan controller is a MATRIX system (Omicron), while the frequency shift $\Delta f$ is detected using an easyPLL Plus phaselocked loop detector from NanoSurf (Liestal, Switzerland). All experiments are carried out under ultra-high vacuum conditions (base pressure $<1 \times 10^{-10}$ mbar) at room temperature. No temperature stabilization inside the laboratory is used. Ar-sputtered n-doped silicon cantilevers (type PPP $\mathrm{NCH}$ ) from NanoWorld (Neuchâtel, Switzerland) with resonance frequencies around $300 \mathrm{kHz}$ are excited to oscillation amplitudes of about $10 \mathrm{~nm}$. For the atom-tracking module, an SR830 lock-in amplifier from Stanford Research Systems (Sunnyvale, CA, USA) is used. Calcite $\mathrm{CaCO}_{3}(10 \overline{1} 4)$ surfaces are prepared by in situ cleaving ${ }^{34}$ and annealing. Further details of the surface and the NC-AFM contrast formation have recently been discussed elsewhere. ${ }^{35}$

First, we characterize the quality of the high-voltage signals based on the results presented in Figure 3. In a first step,

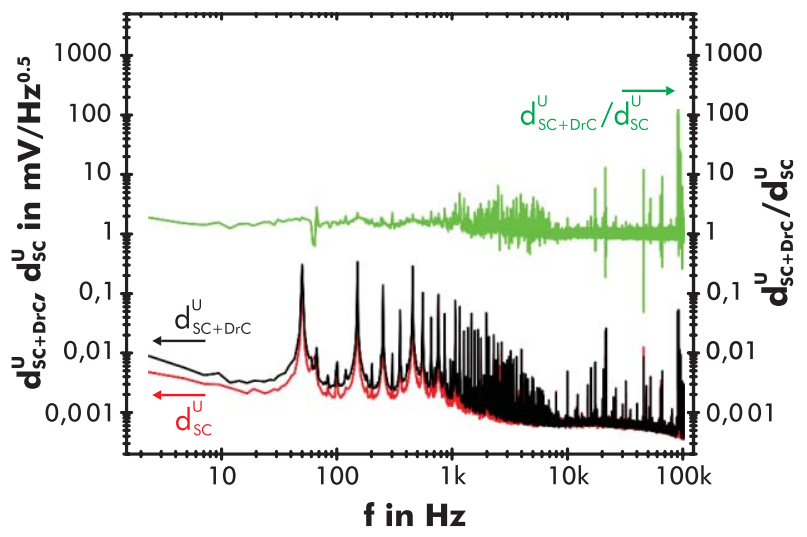

\begin{tabular}{l|cc}
\hline \hline $\mathrm{BW}$ & $\Delta x_{\mathrm{SC}}^{\mathrm{RMS}}$ & $\Delta x_{\mathrm{SC}+\mathrm{DrC}}^{\mathrm{RMS}}$ \\
\hline $100 \mathrm{~Hz}$ & $6.7 \mathrm{pm}$ & $12.5 \mathrm{pm}$ \\
$1 \mathrm{kHz}$ & $13.1 \mathrm{pm}$ & $25.4 \mathrm{pm}$ \\
$10 \mathrm{kHz}$ & $13.7 \mathrm{pm}$ & $26.3 \mathrm{pm}$ \\
$50 \mathrm{kHz}$ & $14.1 \mathrm{pm}$ & $26.6 \mathrm{pm}$ \\
$100 \mathrm{kHz}$ & $14.5 \mathrm{pm}$ & $34.4 \mathrm{pm}$ \\
\hline \hline
\end{tabular}

FIG. 3. (Color online) Noise measurement on the high-voltage $X^{+}$output signal of the original scan controller $\left(d_{\mathrm{SC}}^{\mathrm{U}}\right.$, left scale) and of the combined scan controller and drift compensation system $\left(d_{\mathrm{SC}+\mathrm{DrC}}^{\mathrm{U}}\right.$, left scale). The ratio $d_{\mathrm{SC}+\mathrm{DrC}}^{\mathrm{U}} / d_{\mathrm{SC}}^{\mathrm{U}}$ is referenced to the right scale. In addition, the table lists the piezo dislocation noise for different piezo bandwidths. 

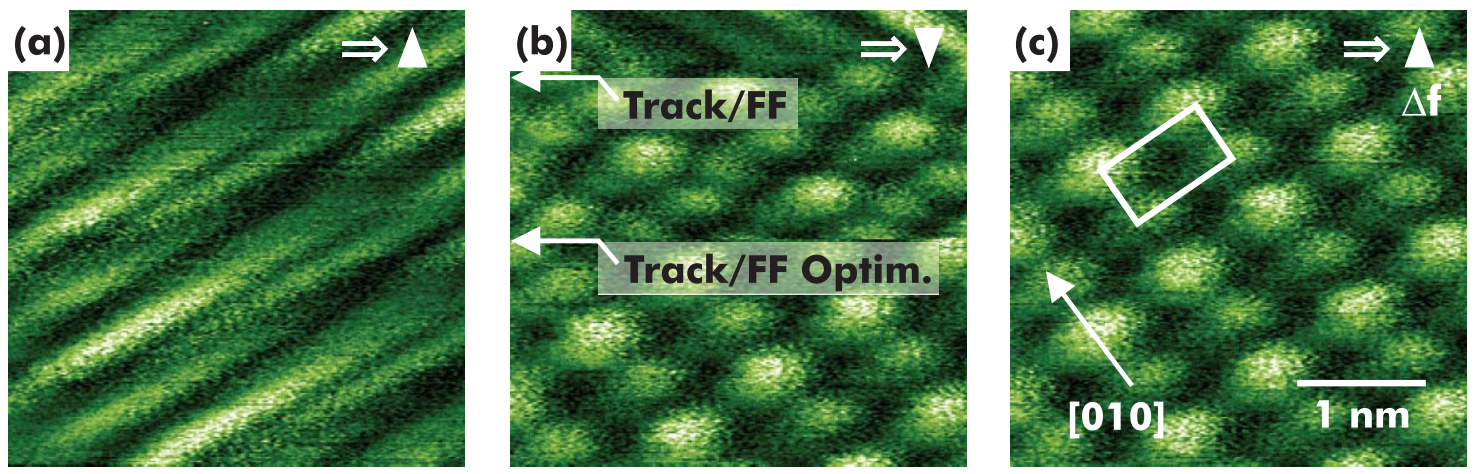

FIG. 4. (Color online) Basic tracking and feed-forward functionality: In (a), large thermal drift is present while imaging the surface. Huge deformations of the imaged surface unit cell are apparent. In (b), the imaging is paused and by using the atom-tracking and feed-forward techniques, drift is measured and compensated ("Track/FF"). The drift parameters were optimized in a second tracking step ("Track/FF Optim."). With compensated drift, distortion-free imaging as presented in (c) is possible for a short time span after tracking.

a spectrum analyzer is connected to the $X^{+}$channel of the scan controller, measuring the signal relative to ground. The noise amplitude spectral density $d_{\mathrm{SC}}^{\mathrm{U}}$ is presented as the red solid curve and despite a few peaks at $50 \mathrm{~Hz}$ and its multiples, for the scan controller signal the spectral amplitudes remain well below $1 \mathrm{mV} / \sqrt{\mathrm{Hz}}$. In a second step, we connect our summation circuitry to the scan controller output and analyze the $X^{+}$signal after the summation circuitry. The respective data $d_{\mathrm{SC}+\mathrm{DrC}}^{\mathrm{U}}$ indicates a slightly increased noise level, which is directly evident from the quotient $d_{\mathrm{SC}+\mathrm{DrC}}^{\mathrm{U}} / d_{\mathrm{SC}}^{\mathrm{U}}$ (right scale in Fig. 3). For the relevant low-frequency regime, we observe an increase by a factor of about two and, additionally, in the upper $\mathrm{kHz}$ regime peaks evolve, which are most likely caused by the DC/DC converters. From the electronic noise we determine the root mean square displacement of the piezo ("piezo dislocation noise") by integrating the power spectral density over different bandwidths and multiplying by the piezo sensitivities. The values for the SC and the combined scan controller and drift compensation system are given in Figure 3. The other output channels have also been checked and yield a comparable behavior (not shown). Due to an increased piezo sensitivity, however, the dislocation noise in the $\mathrm{Z}$ channel is smaller by about a factor of two. As the bandwidth of piezo scanners is usually set to less than $100 \mathrm{kHz}$, the corresponding value has to be regarded as an upper limit for the dislocation noise.

Second, we investigate the basic drift compensation functionality of the tracking and feed-forward modules using a single atom on the $\mathrm{CaCO}_{3}(10 \overline{1} 4)$ surface as reference for the tracking procedure. Figure 4(a) shows an atomically resolved image of the surface with typical distortions present due to large thermal drift. Although the interaction regime is suitable for atomic resolution, single atoms are hardly revealed as their circular shape is elongated to stripes. In Figure 4(b), the raster movement is paused after scanning a few lines from top of the image. During this pause, tracking of a single surface atom is performed and the drift velocity vector is determined from the feedback data. The velocities are used in the feed-forward routine to compensate for linear drift and, consequently, distortion-free imaging at the atomic scale is possible, as presented in Figure 4(c). The precision of the drift velocity measurement depends on the tracking duration and, as present in the middle part of Figure 4(b), a residual distortion is present if the time span selected is too small. Therefore, the feed-forward parameters are optimized in a second tracking step as indicated in Figure 4(b). With the thermal drift minimized, we measure the unit cell dimensions to be $10.0(2) \times 8.1(2) \AA$ in excellent agreement with the crystallographic bulk data ${ }^{36}$ of $10.0 \times 8.1 \AA$.

Third, we focus on the long-term drift characteristics of the microscope in addition to the tip-positioning precision of the atom-tracking module. Again, a single surface atom on a $\mathrm{CaCO}_{3}(10 \overline{1} 4)$ surface is tracked for a period of about 75 min. The corresponding data are presented in Figure 5 in the form of path-time diagrams. Each curve represents the feature position along the $\mathrm{X}, \mathrm{Y}$, and $\mathrm{Z}$ directions, respectively. As an immobile feature is tracked, each curve directly describes the relative drift movement between tip and sample. The non-linear behavior, which is well-known from roomtemperature conditions, is most pronounced for the $\mathrm{Z}$ movement, which is even non-monotonic. For example, we determine the approximated linear drift for the $\mathrm{X}$ direction from a linear least-squares fit to be about $-117 \mathrm{pm} / \mathrm{min}$, while the curvature is calculated from a quadratic least-squares fit to be $\sim 1.18 \mathrm{pm} / \mathrm{min}^{2}$. Predicting the long-term drift behavior

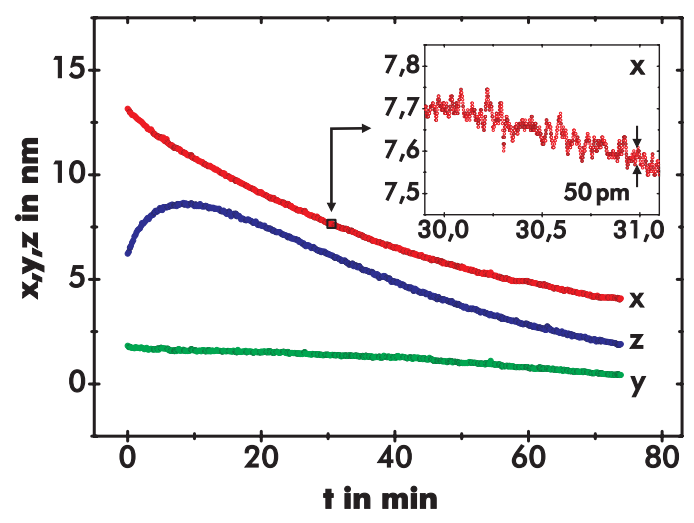

FIG. 5. (Color online) Drift characteristics and positioning precision of our drift-compensation system. Here, a surface species on the $\mathrm{CaCO}_{3}(10 \overline{1} 4)$ surface is used as the reference, the drift is measured over about $75 \mathrm{~min}$. Nonlinear drift behavior is directly evident and for the $Z$ channel, the drift is even non-monotonic. 
would be a simple task if these values were constant with time. This, however, is usually not the case in roomtemperature experiments. Calculating the root mean square dislocation noise from the data, we determine a tip positioning precision better than $50 \mathrm{pm}$ as shown in the inset of Figure 5.

The positioning precision is somewhat larger than the tip dislocation noise induced by the electronic circuitry as analyzed before. Most likely, this is a result of the large bandwidth settings for the lateral feedback loops. The instrumental limit of the tip positioning precision for the present system is $8 \mathrm{pm}$ in lateral and $2.6 \mathrm{pm}$ in vertical direction as determined from the digital-analog conversion, signal amplification, and piezo sensitivities.

\section{PROTOCOL FOR 3D DATA ACQUISITION WITH NON-LINEAR DRIFT COMPENSATION}

Abe et al. have presented a protocol for reproducible spectroscopy experiments at room temperature by pausing and resuming the atom tracking in between data acquisition. ${ }^{30}$ With this protocol, they have been able to reproducibly obtain precise $\Delta f(z)$ data of a $\mathrm{Sn}$ substitutional adatom on a $\mathrm{Si}(111)$ surface $^{30}$ and, subsequently, used this protocol for chemical identification of single adatoms. ${ }^{5}$ We extend this protocol to acquire a complete 3D data set by an automated procedure yielding dense 3D data under conditions of non-linear drift. The timing for the different steps followed during the drift compensation and data acquisition is depicted in Figure 6(b), while Figure 6(a) depicts the tip trajectory.
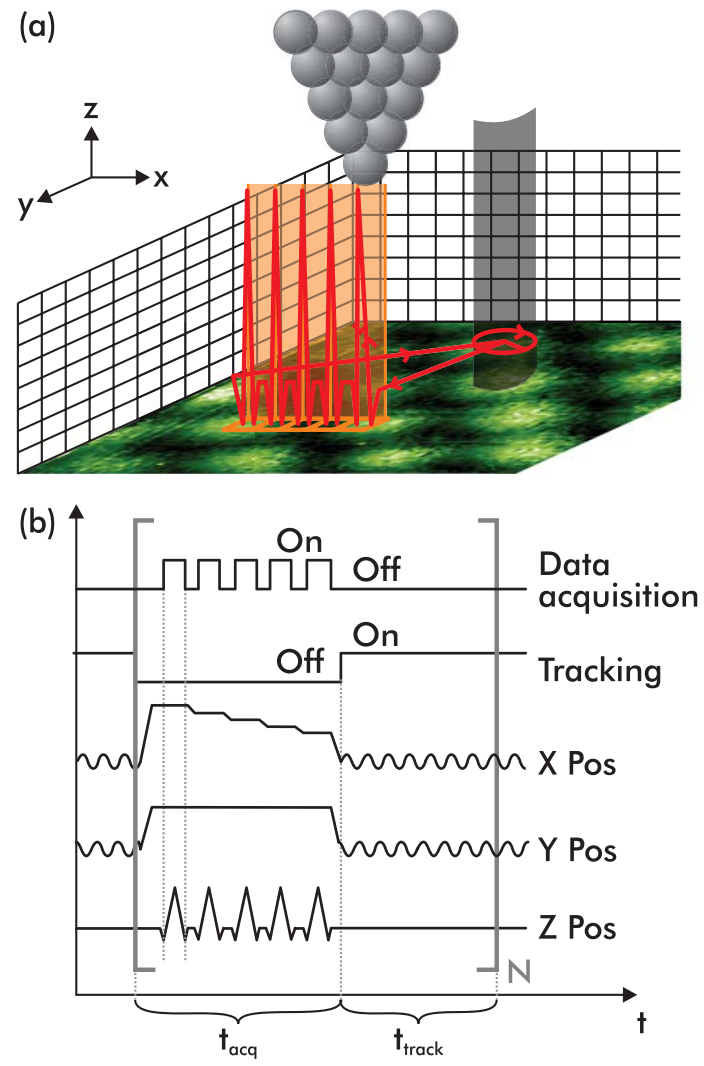

FIG. 6. (Color online) Protocol for the 3D data acquisition under large drift conditions using a point of origin defined by the tracking system. (a) sketches the tip trajectory while (b) presents the timing diagram.
In a first step, atom tracking is started and a single surface species is tracked for a short time span. From this tracking data, the instantaneous linear drift in all three spatial directions is compensated. However, this compensation is not capable of handling the non-linear drift present over a longer time span unless the precise drift behavior is known - which is usually not the case. After the initial drift compensation, the 3D data acquisition procedure itself is started. Here, we discuss the sampling in the form of $\Delta f(z)$ curves, but recording single images (or parts of images) is also possible. For each data acquisition step (as depicted by a bracket in Figure 6(b)), the tracking is first paused by stopping the lateral feedback loops and the tip dithering. The tip is brought to the center of the dithering circle. At this time, the reference point of the tip is well-defined with respect to the previous tracking position and remains valid with high precision for a short time interval.

As the next step, $\Delta f(z)$ data are recorded systematically by relocating the tip to the desired grid position and executing the $\Delta f(z)$ curve measurement, which moves the tip in the $\mathrm{Z}$ direction while sampling data. ${ }^{38}$ Although several curves can be sampled during a single pause, the duration $t_{\text {acq }}$ of data acquisition is limited (as will be analyzed in detail later). To account for the non-linearity of the drift and the consequent tip misplacement, the absolute position needs to be redefined in a next step. Hence, the tip is moved back to the position where the tracking was paused and, finally, the tracking is restarted by dithering the tip and reactivating the feedback loops. During a time span of $t_{\text {track }}$, the tracking module centers the tip on top of the selected protrusion and, thus, non-linear drift contributions are automatically compensated. It should be noted that short delays in the order of $0.1 \mathrm{~s}$ are introduced after each tip relocation to compensate for piezo scanner creep. The procedure is repeated $N$ times until all data points are sampled, as depicted in Figure 6(b).

Due to the non-linearity of the drift, an upper limit for the acquisition time $t_{\text {aqc }}$ exists. We estimate this limit from the relation

$$
r_{\text {drift }}\left(t_{\text {acq }}\right)-r_{\text {drift }}(0)<\Delta g
$$

where $r_{\text {drift }}(t)$ is the misplacement at time $t$ due to thermal drift and $\Delta g$ is the desired positioning precision. With this equation, we constrain the total tip-sample misplacement occurring during the acquisition step to be smaller than $\Delta g$, a quantity which can be chosen according to the grid or surface unit cell size. Assuming a quadratic behavior $r(t)$ $=1 / 2 a_{\text {drift }} t^{2}$ with an acceleration $a_{\text {drift }}$, we find an upper limit for the acquisition time $t_{\text {acq }}$ :

$$
t_{\text {acq }}<\sqrt{\frac{2 \Delta g}{a_{\text {drift }}} .}
$$

With a desired precision of $\Delta g=1 \mathrm{pm}$ and a curvature of $a_{\mathrm{drift}}=1 \mathrm{pm} / \mathrm{min}^{2}$, a maximum acquisition time of $t_{\text {acq }}=85 \mathrm{~s}$ would be possible until the tip is misplaced by more than the expected precision. However, this calculation is performed with the assumption of zero drift at $t=0$. This can only be assured, if the drift vector for the feedforward module is updated frequently. This update interval $t_{\text {update }}$ is also dependent on the curvature $a_{\text {drift }}$. To give an 


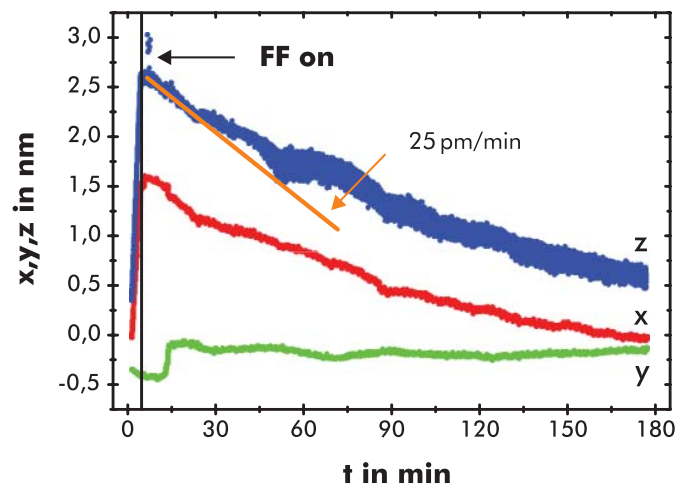

FIG. 7. (Color online) Drift characteristics during the 3D data acquisition on a calcite $\mathrm{CaCO}_{3}(10 \overline{1} 4)$ surface. After the feed-forward (FF) is activated with the drift velocities determined by the tracking, the residual drift is $25 \mathrm{pm} / \mathrm{min}$ at maximum as indicated by the orange line.

example, after $10 \mathrm{~min}$ the residual drift is in the order of $v_{\text {drift }}=a_{\text {drift }} \times 10 \mathrm{~min}=10 \mathrm{pm} / \mathrm{min}$. Therefore, by defining a maximum tolerable drift $v_{\text {drift }}$, an upper limit for the update interval $t_{\text {update }}$ is directly obtained.

Another constraint for the acquisition time $t_{\text {acq }}$ arises from the feature dimensions: As the atom-tracking technique always recenters the tip to the nearest extremum, it might lose the point of origin if the mislocation is larger than the feature dimension. On an atomic lattice, this happens if the mislocation is larger than the lattice constant $a$ and, therefore, $\Delta g \ll a$ has to be ensured for this case.

\section{ACQUISITION PROTOCOL DEMONSTRATION}

We demonstrate the protocol on a calcite $\mathrm{CaCO}_{3}(10 \overline{1} 4)$ surface at the atomic scale. During the $3 \mathrm{~h}$ of data acquisi- tion, a complete 3D force map above the surface is taken. The initial drift is found to be $525 \mathrm{pm} / \mathrm{min},-23 \mathrm{pm} / \mathrm{min}$, and $754 \mathrm{pm} / \mathrm{min}$ in the $\mathrm{X}, \mathrm{Y}$, and $\mathrm{Z}$ directions, respectively, and compensated by the feed-forward module. The origin for this drift might be thermal material relaxation, though the temperature gradient measured at the sample stage has been as small as about $8 \mathrm{mK} / \mathrm{h}$. The positioning data, which are the actuating variables from the tracking feedback loops, are presented in Figure 7 and directly represent the residual drift movements. During the experiment, drift velocities are optimized periodically to ensure residual drift velocities smaller than $25 \mathrm{pm} / \mathrm{min}$. This parameter update is performed about every $15 \mathrm{~min}$ as this time span is expected to meet our requirements for the positioning precision, as discussed before.

A total of $7225 \Delta f(z)$ curves are acquired on a grid of $85 \times 85$ pixel, representing $1.74 \times 1.74 \mathrm{~nm}^{2}$ of the surface. In the vertical direction, a total of 500 pixel along $2.18 \mathrm{~nm}$ are sampled per $\mathrm{Z}$ forward and backward tip movement while the sampling time per point is chosen as $1 \mathrm{~ms}$. A total of 17 curves are acquired during each acquisition interval of $t_{\mathrm{acq}} \sim 18.5 \mathrm{~s}$. The tracking is restarted for $t_{\text {track }}=5 \mathrm{~s}$ in-between the acquisition of 17 curves to recenter the tip at the point of origin. The acquisition and tracking steps are repeated $N=425$ times during a total measurement time of about $3 \mathrm{~h}$. For the tracking procedure, a surface depression was chosen while the dithering amplitude amounts to $A_{\mathrm{x}, \mathrm{y}}=1.2 \AA$ at a frequency of $f_{\mathrm{x}, \mathrm{y}}=20 \mathrm{~Hz}$. The tracking position is marked by a circle in Figure 8(e).

In Figure 8, different representations of the $85 \times 85$ $\times 500$ pixel data space are depicted. With a 3D data set (Fig. 8(a)) available, it is straightforward to extract lateral constant-height $\mathrm{X}-\mathrm{Y}$ images at different $\mathrm{Z}$ positions (Figs. 8(e) and 8(f)), vertical X-Z (Fig. 8(c)), or Y-Z
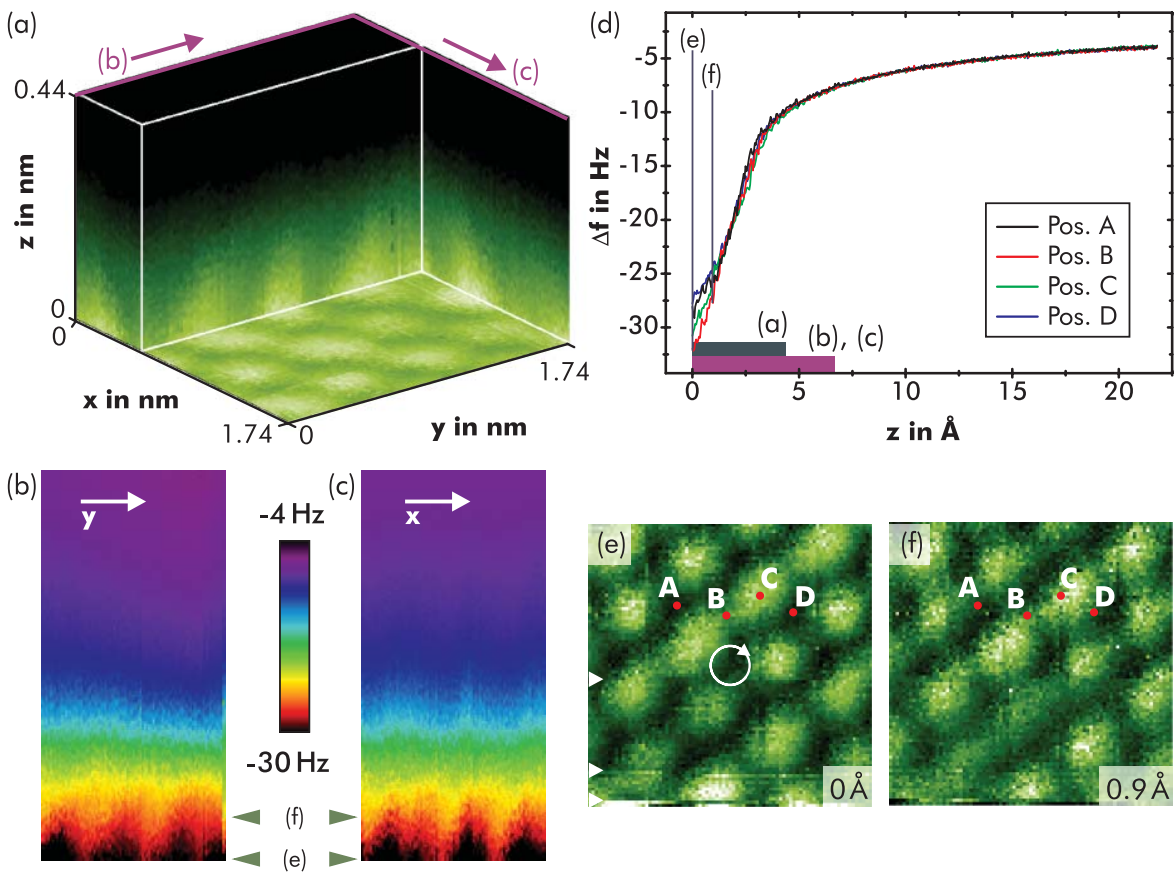

FIG. 8. (Color online) Different representations of the force field above $\mathrm{CaCO}_{3}(10 \overline{1} 4)$ : (a) presents a cropped 3D view of the data acquired. Planes along different axes are extracted and presented in (b), (c), (e), and (f). Furthermore, it is possible to extract $\Delta f(z)$ curves from different surface sites as depicted in (d). The trajectory of the circular tip movement is depicted in (e). 
(Fig. 8(b)) slices as well as $\Delta f(z)$ spectroscopy curves (Fig. 8(d)). From the latter distance-dependent data it is possible to calculate the interaction force and potential using the formulae presented in Ref. 37. It should be noted that all presented data are raw data and have not been enhanced by smoothing or fitting procedures. Most notably, our protocol does not demand any subsequent drift correction as proposed elsewhere ${ }^{20}$ and, therefore, does not need any assumptions regarding the sample system and the AFM contrast.

In the acquired data, we can identify few tip changes as marked by white triangles in Figure 8(e). Though tip changes are usually most undesirable in AFM experiments, here they demonstrate the stability of our drift compensation protocol. The tracking module is capable of automatically recentering the tip to the predefined surface site regardless of the tip termination.

\section{CONCLUSIONS}

In conclusion, we present and characterize a newly developed and highly flexible drift-compensation system. The system utilizes atom-tracking and feed-forward principles and a tip positioning precision of better than $50 \mathrm{pm}$ can be achieved. It is well-suited for operation in a large drift environment, as typically present when performing experiments at room temperature. With this system, we have developed and implemented a scan protocol for 3D data acquisition capable of handling large and non-linear drift. The scan protocol eliminates the problems arising from severe thermal drift and only leaves the tip stability as the main limitation. Furthermore, it does not need any subsequent drift correction of the acquired data. As a performance test, we present 3D data from a calcite $\mathrm{CaCO}_{3}(10 \overline{1} 4)$ surface with atomic resolution.

\section{ACKNOWLEDGMENTS}

The authors gratefully acknowledge most helpful discussions with H. H. Pieper, S. Bahr, L. Tröger, J. Lübbe, O. Lohre, and B. Lemme from the Universität Osnabrück and with D. Sawada and K. Morita from Osaka University. Assistance from J. Köble (Omicron) with the MATE scripting system is highly appreciated. We are grateful to P. Moriarty from the University of Nottingham for his valuable contribution.

This work has been supported by the German Research Foundation (DFG) through the DFG project KU1980/4-1 and the DFG-JSPS agreement (446 JAP 113/337/322/0-1).

${ }^{1}$ F. J. Giessibl, Rev. Mod. Phys. 75, 949 (2003).

${ }^{2}$ C. Barth, A. S. Foster, C. R. Henry, and A. L. Shluger, Adv. Mater. 23, 477 (2011).

${ }^{3}$ M. Z. Baykara, T. C. Schwendemann, E. I. Altman, and U. D. Schwarz, Adv. Mater. 22, 2838 (2010).
${ }^{4}$ L. Gross, F. Mohn, N. Moll, P. Liljeroth, and G. Meyer, Science 325, 1110 (2009).

${ }^{5}$ Y. Sugimoto, P. Pou, M. Abe, P. Jelínek, R. Pérez, S. Morita, and Ó. Custance, Nature (London) 446, 64 (2007).

${ }^{6}$ Y. Sugimoto, T. Namikawa, K. Miki, M. Abe, and S. Morita, Phys. Rev. B 77, 195424 (2008).

${ }^{7}$ B. Such, T. Glatzel, S. Kawai, S. Koch, and E. Meyer, J. Vac. Sci. Technol. B 28, C4B1 (2010).

${ }^{8}$ K. Ruschmeier, A. Schirmeisen, and R. Hoffmann, Phys. Rev. Lett. 101, 156102 (2008).

${ }^{9}$ K. Ruschmeier, A. Schirmeisen, and R. Hoffmann, Nanotechnology 20, 264013 (2009).

${ }^{10}$ A. Schirmeisen, D. Weiner, and H. Fuchs, Phys. Rev. Lett. 97, 136101 (2006).

${ }^{11}$ S. Kawai, T. Glatzel, S. Koch, A. Baratoff, and E. Meyer, Phys. Rev. B 83, 035421 (2011).

${ }^{12}$ A. Schwarz, H. Hölscher, S. M. Langkat, and R. Wiesendanger, Scanning Tunneling Microscopy/Spectroscopy and Related Techniques: 12th International Conf., 696, 68 (2003).

${ }^{13}$ H. Hölscher, S. M. Langkat, A. Schwarz, and R. Wiesendanger, Appl. Phys. Lett. 81, 4428 (2002).

${ }^{14}$ S. M. Langkat, H. Hölscher, A. Schwarz, and R. Wiesendanger, Surf. Sci. 527, 12 (2003).

${ }^{15}$ M. Heyde, G. H. Simon, H. P. Rust, and H. J. Freund, Appl. Phys. Lett. 89, 263107 (2006).

${ }^{16}$ B. J. Albers, T. C. Schwendemann, M. Z. Baykara, N. Pilet, M. Liebmann, E. I. Altman, and U. D. Schwarz, Nat. Nanotechnol. 4, 307 (2009).

${ }^{17}$ D. A. Braun, D. Weiner, B. Such, H. Fuchs, and A. Schirmeisen, Nanotechnology 20, 264004 (2009).

${ }^{18}$ D. A. Braun, G. Langewisch, H. Fuchs, and A. Schirmeisen, J. Vac. Sci. Technol. B 28, C4B6 (2010).

${ }^{19}$ M. Ashino, D. Obergfell, M. Haluska, S. H. Yang, A. N. Khlobystov, S. Roth, and R. Wiesendanger, Nanotechnology 20, 264001 (2009).

${ }^{20}$ B. J. Albers, T. C. Schwendemann, M. Z. Baykara, N. Pilet, M. Liebmann, E. I. Altman, and U. D. Schwarz, Nanotechnology 20, 264002 (2009).

${ }^{21}$ P. Rahe, R. Bechstein, and A. Kühnle, J. Vac. Sci. Technol. B 28, C4E31 (2010).

${ }^{22}$ T. Fukuma, Y. Ueda, S. Yoshioka, and H. Asakawa, Phys. Rev. Lett. 104, 016101 (2010).

${ }^{23}$ T. Fukuma, M. Kimura, K. Kobayashi, K. Matsushige, and H. Yamada, Rev. Sci. Instrum. 76, 053704 (2005).

${ }^{24}$ D. W. Pohl and R. Möller, Rev. Sci. Instrum. 59, 840 (1988).

${ }^{25}$ B. S. Swartzentruber, Phys. Rev. Lett. 76, 459 (1996).

${ }^{26}$ B. S. Swartzentruber, A. P. Smith, and H. Jónsson, Phys. Rev. Lett. 77, 2518 (1996)

${ }^{27}$ X. R. Qin, B. S. Swartzentruber, and M. G. Lagally, Phys. Rev. Lett. 85, 3660 (2000).

${ }^{28}$ M. Krueger, B. Borovsky, and E. Ganz, Surf. Sci. 385, 146 (1997).

${ }^{29}$ E. Hill, B. Freelon, and E. Ganz, Phys. Rev. B 60, 15896 (1999).

${ }^{30}$ M. Abe, Y. Sugimoto, Ó. Custance, and S. Morita, Nanotechnology 16, 3029 (2005).

${ }^{31}$ M. Abe, Y. Sugimoto, Ó. Custance, and S. Morita, Appl. Phys. Lett. 87, 173503 (2005).

${ }^{32}$ M. Abe, Y. Sugimoto, T. Namikawa, K. Morita, N. Oyabu, and S. Morita, Appl. Phys. Lett. 90, 203103 (2007).

${ }^{33}$ Winavr project, URL http://winavr.sourceforge.net.

${ }^{34}$ L. Tröger, J. Schütte, F. Ostendorf, A. Kühnle, and M. Reichling, Rev. Sci. Instrum. 80, 063703 (2009).

${ }^{35}$ J. Schütte, P. Rahe, L. Tröger, S. Rode, R. Bechstein, and A. Kühnle, Langmuir 26, 8295 (2010).

${ }^{36}$ S. A. Markgraf and R. J. Reeder, American Mineralogist 70, 590 (1985).

${ }^{37}$ J. E. Sader and S. P. Jarvis, Appl. Phys. Lett. 84, 1801 (2004).

${ }^{38}$ Here, the MATE scripting environment (Omicron) is used to synchronize the data acquisition with the tracking steps. However, a trigger signal could be used for the synchronization as well. 